\title{
Gastric Outlet Obstruction as an Aggravation of Peutz- Jeghers Syndrome: Case Report
}

\author{
Marcelo Zamorano ${ }^{1,2 *}$, Bruno Baluarte ${ }^{1}$, Camila Del Valle $^{2}$, Alexandra Deichler ${ }^{1}$, Edgardo \\ Mancilla $^{1}$, Rafaella V Zárate ${ }^{3}$ and Duxan Arancibia ${ }^{3 *}$ \\ ${ }^{1}$ Unit of Surgery, Faculty of Medicine and Dentistry, University of Antofagasta, Chile \\ ${ }^{2}$ Digestive Surgery Team, Helping Hands Foundation, Antofagasta, Chile \\ ${ }^{3}$ Department of Medical Sciences, Faculty of Medicine and Dentistry, University of Antofagasta, Chile
}

*Corresponding author: Marcelo Zamorano, Surgery Unit, Faculty of Medicine and Dentistry, University of Antofagasta, Antofagasta, Chile and Duxan Arancibia, Department of Medical Sciences, Faculty of Medicine and Dentistry, University of Antofagasta, Chile

\section{ARTICLE INFO}

Received: 慧 August 14, 2021

Published: 幽 August 31, 2021

Citation: Marcelo Zamorano, Bruno Baluarte, Camila Del Valle, Alexandra Deichler, Duxan Arancibia, et al., Gastric Outlet Obstruction as an Aggravation of Peutz-Jeghers Syndrome: Case Report. Biomed J Sci \& Tech Res 38(3)-2021. BJSTR. MS.ID.006160.

Abbreviations: PJS: Peutz-Jeghers Syndrome; G00: Gastric Outlet Obstruction; CT: Computed Tomography; VAS: Visual Analog Scale

\begin{abstract}
The Peutz-Jeghers Syndrome (PJS) is a rare autosomal dominant disease characterized by macules of mucocutaneous pigmentation and hamartomatous intestinal polyps. Some signs of the PJS appear in childhood, usually with abdominal pain. In some cases, patients can develop complications. In this article, we report a case of a patient with PJS that reached the emergency room after presenting a concomitant gastric outlet obstruction (GOO). The patient underwent through an endoscopic polypectomy, followed by a laparotomy and an intraoperative enteroscopy. The symptoms were controlled and the extraction of hamartomatous intestinal polyps were achieved, culminating with an intestinal segment resection with muscle atrophy and posterior gastrojejunostomy. There is evidence of PJS cases related to other conditions that developed abnormal scenarios such as biliary obstruction or cecal appendix polyps. However, the association between PJS and GOO and a concomitant delimited intestinal aperistalsis has not been reported. Thus, this case report shows the possible association between the PJS and GOO, whose cause is the intraluminal occupation of the duodenum by polyps and the synergy of the symptoms given by the atrophy of the jejunum muscle.
\end{abstract}

Keywords: Peutz-Jeghers Syndrome; Peutz- Jeghers Aggravations; Gastric Outlet Obstruction

\section{Background}

The proliferation of polyps or polyposis is mostly associated with the elderly. However, a small proportion of cases is related to hereditary disorders, including the Peutz Jeghers Syndrome (PJS), a rare disease with an estimated incidence of 1 in 25,000 to 300,000 live births [1]. PJS is based on three clinical pillars:

a. The appearance of melanic mucocutaneous pigmentation, in the form of small macules of 1 to $5 \mathrm{~mm}$ around the mouth, eyes, anus, fingers, and toes [2]. b. The appearance of 1 to 20 hamartomatous polyps per intestinal segment generally found in the small intestine, most frequently in the jejunum and followed by the ileum and duodenum [3]. These polyps are formed by glandular epithelium supported by an arboriform proliferation of smooth muscle. The smooth muscle bands fan out into the polyp head and thin as they advance to the surface [3].

c. The autosomal dominant hereditary character. Nevertheless, it should be noted that cases of PJS without a hereditary 
association have also been reported, the so-called "sporadic PJS".

d. The polyps found in PJS are commonly present in early adulthood. One-third of affected people experience symptoms during the first ten years of life, with intermittent abdominal pain being the most common of them [4]. This pathology is believed to originate from the germline mutation of the serine/ threonine kinase 11 (SKT11) gene. It has been reported that patients who suffer from this mutation have a risk between 9 to 15 times greater than the general population of suffering intestinal cancer [5,6], and to a lesser extent, extraintestinal cancer, specifically the lung, breast, uterus, testicle, and ovary cancer [7]. Moreover, several studies have confirmed a high association between the genetic alteration in STK11 and the oncological pathology.

The management of PJS begins with active endoscopic surveillance such as upper gastrointestinal endoscopy, video capsule endoscopy, and colonoscopy, searching for polyps. Other study alternatives are magnetic resonance enterography or computed tomography and barium follow-up that could be more precise. Nevertheless, the latter is limited by radiation exposure and should be avoided in children [8-10]. The main complications of this syndrome are anemia, intussusception, and intestinal obstruction; however, despite the intestinal obstruction being a prevalent complication, the presentation of a PJS with gastric retention syndrome is infrequent. From this premise, the objective of this study was to present the case of a patient with a diagnosis of PJS, who presented a duodenal polyp that obstructed the lumen. This obstruction was manifested by a gastric outlet obstruction (GOO) and simultaneously with the investigation of a hypotonic and a peristaltic segment of the proximal jejunum. In this study, we report the possible association between the PJS and GOO, which so far, there are no reports of an SPJ case with a similar presentation.

\section{Case Report}

A 25-year-old female patient with a morbid history of PeutzJeghers Syndrome, without previous complications and without follow-up, goes to the emergency department due to symptoms of one month of evolution and intensified during the last two days. The patient consults for pain in the epigastrium, periumbilical irradiation of progressive intensity reaching a visual analog scale (VAS) of 10/10, colic type and associated with explosive vomiting and a loss of $7 \mathrm{~kg}$ of weight in the last month. A physical examination highlighted: allergic facial, dehydrated mucous membranes and decreased turgor. The patient exhibited faint macules on the oral mucosa, for which she underwent dermatological laser treatment (Figures 1A \& 1B). In addition, she presented a soft, depressible, and painless abdomen, without the presence of masses or visceromegaly. A computed tomography (CT) scan of the chest, abdomen, and pelvis was performed, which revealed images suggestive of multiple polyps at the level of the duodenum-jejunal transition and proximal jejunum.

\section{A}

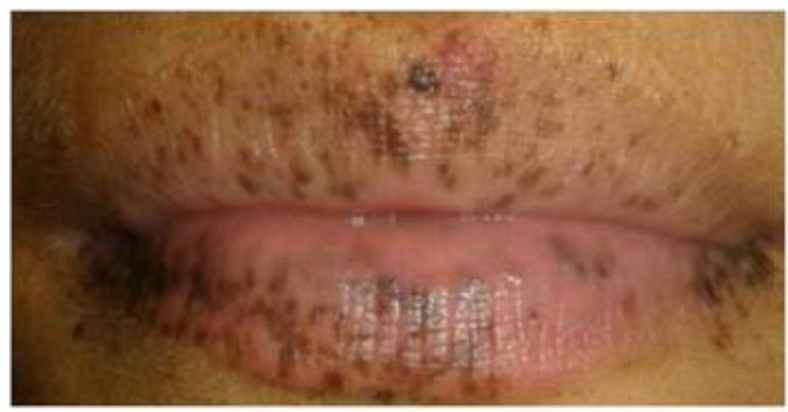

\section{B}

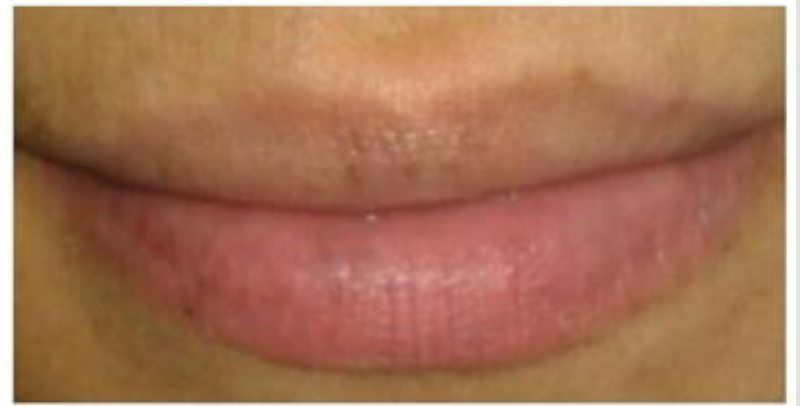

Figure 1:

A. Patient with multiple peri-oral pigmented macules at the time of diagnosis.

B. After laser treatment, the patient shows the disappearance of the macules almost entirely at the time of the physical examination in the Emergency Department.

Also, retention distended stomach was revealed. Considering the symptomatology and the pathological findings in the tomography, it was decided to perform an endoscopic polypectomy in the pavilion. In this procedure, a pedunculated polyp obstructing the intestinal lumen at the level of the third to fourth duodenal portion was found and resected. A rapid biopsy obtained was negative for dysplasia and malignancy (Figure 2). Subsequently, a colonoscopy was performed, which allowed identifying a $10 \mathrm{~mm}$ diameter pedunculated lesion located in the distal sigmoid colon. This lesion exhibited a polypoid appearance with an erythematous 
head, and fibrin remains on its surface. The patient evolved with persistent abdominal pain, intermittent vomiting of lesser quantity, and periodicity. Control computed tomography with oral contrast reported a slowdown in the passage of contrast medium at the level of the proximal jejunum with the presence of polyps that did not completely obstruct the lumen. Based on the results obtained in the follow-up studies, it was decided the re-intervention of the patient employing an exploratory laparotomy and intraoperative enteroscopy.
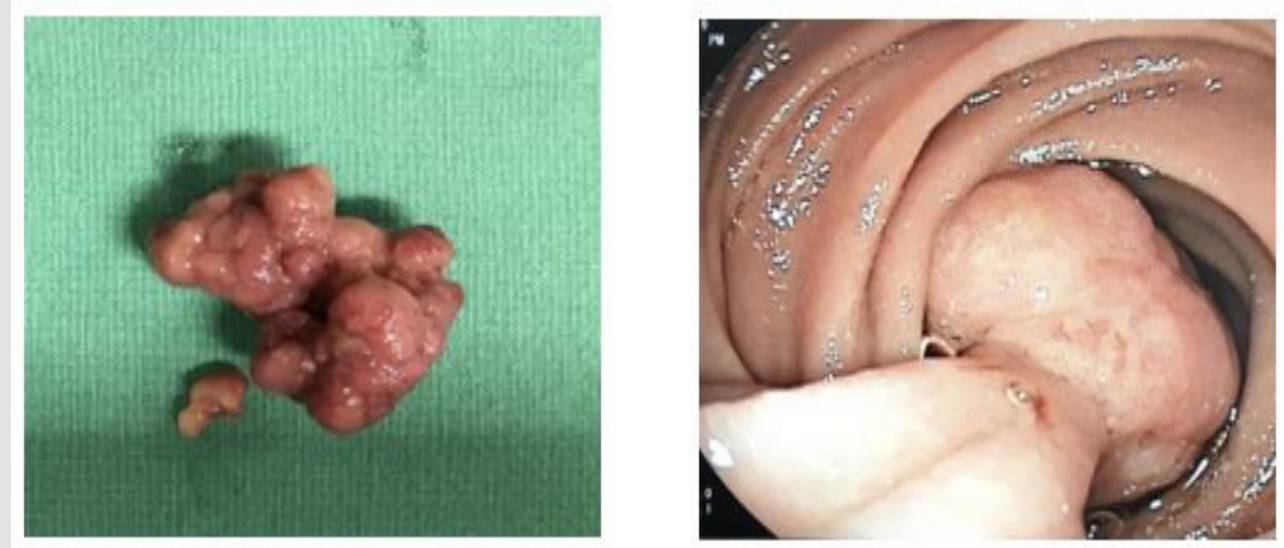

Figure 2: Peutz-Jegher pedunculated hamartomatous polyp, located in the duodenum, completely obstructing the intestinal lumen. This polyp was resected by endoscopic polypectomy.

\section{Results}

During the procedure, a polyp was found at the fourth portion of the duodenum level, and multiple polyps in the distal ileum from 5 to $40 \mathrm{~cm}$ from the ileocecal valve that was extracted with a biopsy forceps. Moreover, the last polyp located $120 \mathrm{~cm}$ from the ileocecal valve was resected endoscopically. None of the polyps found obstructed the entire intestinal lumen. Also, a proximal jejunum area of approximately $15 \mathrm{~cm}$ with aperistalsis was detected. A neostigmine test was performed with local infiltration, confirming abolished peristalsis in the proximal jejunum area. The rest of the small intestine maintained good contractibility. The jejunal segment $(15 \mathrm{~cm})$ was resected with its mesentery (it was sent for a deferred histopathological study) (Figure 3), culminating the intervention with a gastro-jejunal-lateral anastomosis performed without complications. In the histopathological study, a muscle wall thinned was observed, especially at the expense of the internal circular. Even, a loss of muscle fiber density was observed, with connective tissue spaces between them (Figure 4). Also, a degenerative changes of the muscle tissue of the internal circular was visualized, along with loss of muscle fibers (Figure 5). After surgery, the patient showed a favorable evolution and remained hemodynamically stable and afebrile. She presented a progressive decrease in pain, gas elimination, and preserved stools, and she was discharged on the $8^{\text {th }}$ postoperative day. At postoperative control up to 6 months, the patient remained asymptomatic.

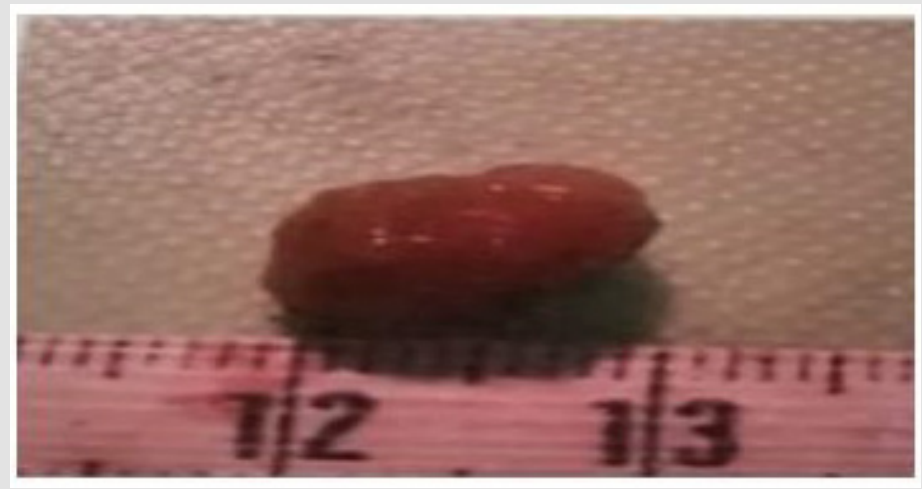

Figure 3: Peutz-Jeghers type hamartomatous polyp resected from the ascending colon and histologically described with a complex polypoid arrangement. This polyp is composed of bands of smooth muscle tissue surrounded by glands without epithelial atypia but with focal cystic hyperplasia and dilatation. 


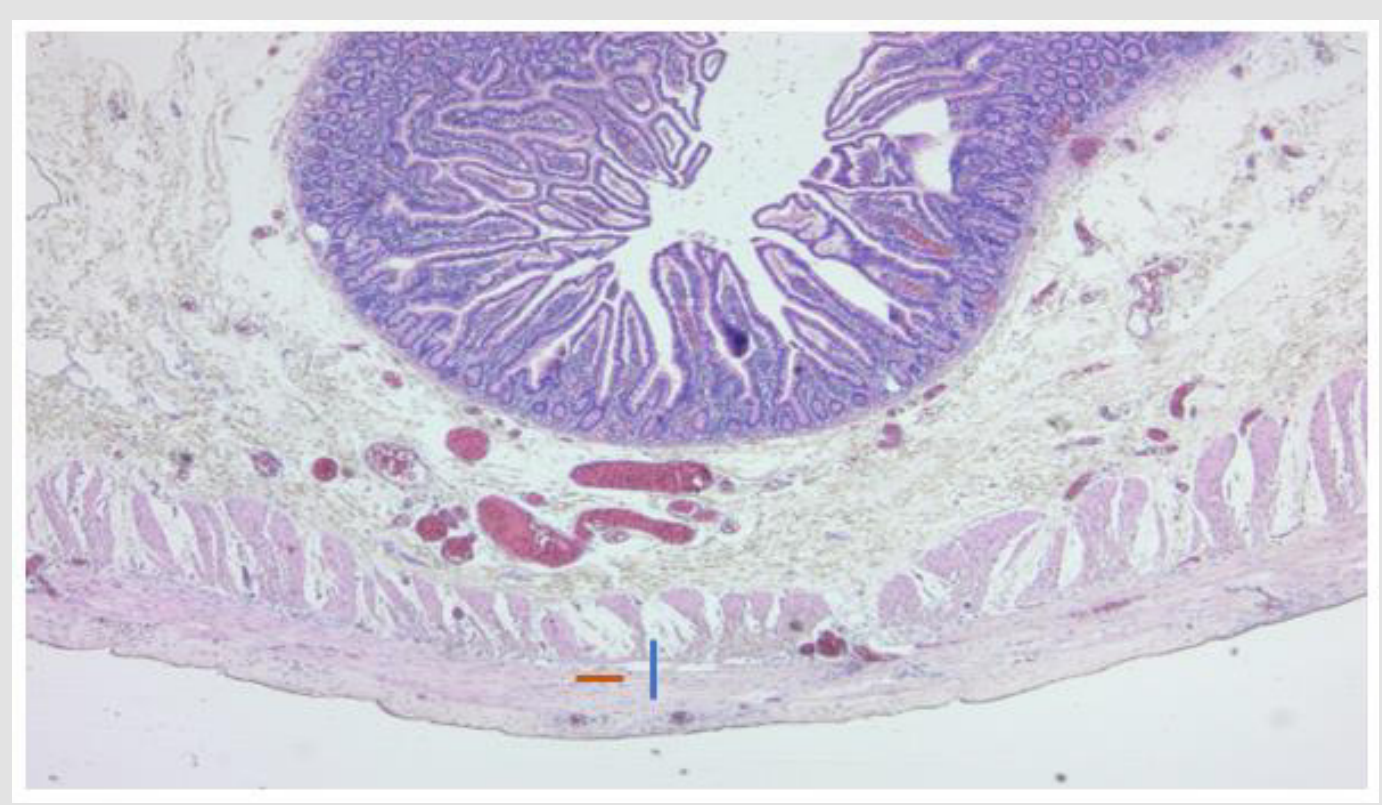

Figure 4: Muscle wall thinned, especially at the expense of the internal circular (blue line). Loss of muscle fiber density is observed, with connective tissue spaces between them (orange line).

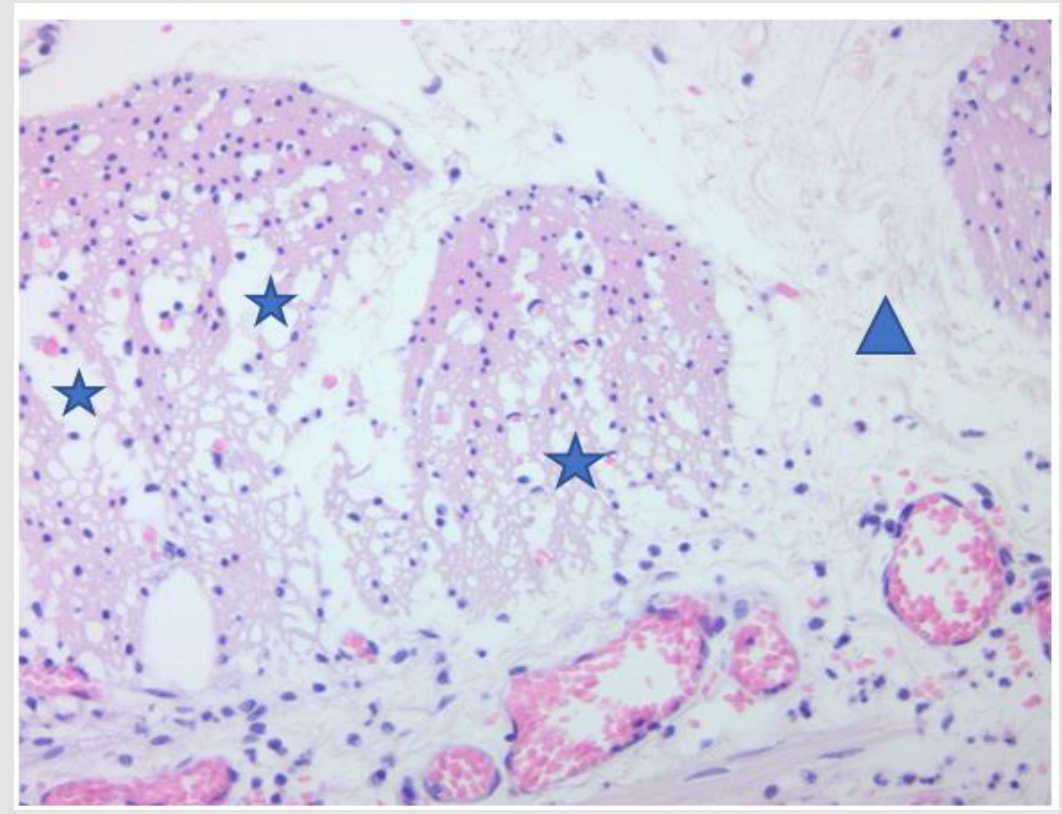

Figure 5: Degenerative changes of the muscle tissue of the internal circular (blue stars), loss of muscle fibers (blue triangle).

\section{Discussion}

Peutz-Jeghers syndrome is an autosomal dominant disease characterized by the finding of multiple hamartomatous polyps in the gastrointestinal tract, mucocutaneous pigmentation, and an increased risk of both gastrointestinal and non-gastrointestinal cancer [11]. Concerning mucocutaneous pigmentation, it is present in more than $95 \%$ of individuals with PJS and is caused by pigment- laden macrophages in the dermis. This pigmentation is usually flat, bluish-gray to brown spots 1 to $5 \mathrm{~mm}$ in size, and generally manifests during the first or second year of life. The size and number of pigmentations increase continuously in the following years and finally clear up after puberty, except for those located in the buccal mucosa [7]. Unfortunately, not all patients may exhibit mucocutaneous pigmentation due to the time of the disease or 
because the patient underwent dermatological laser treatment on the macules. In this treatment, the laser vibration can alter the pigmentary system, and the beams may absorb the dark color of the melanocytes in the pigmented areas, masking this symptom.

On the other hand, polyps appear mainly in the small intestine (in order of prevalence: jejunum, ileum, and duodenum), but they can also be located in the colon or stomach. Histologically, they are defined by a normal epithelium surrounding smooth muscle bundles arranged in a branched or leafy pattern [8]. As far as we know, no other cases were found related to aperistalsis of the small intestine. It seems a feasible theory in the relationship with the atrophy of the smooth muscle presented in the case, the same of the formation of polyps: the thinning of the smooth muscle when they advance to the surface. The mentioned clinical characteristics were manifested in a typical way in our patient. The patient presented numerous peri-oral pigmented macules at the beginning of the disease. Also, multiple polyps appeared predominantly in the small intestine, a common and known feature in this syndrome. The diagnosis of PJS is made clinically in patients with hamartomatous polyps, demonstrated by histology if two of the following three conditions are present: positive family history with an autosomal dominant inheritance pattern, mucocutaneous hyperpigmentation and/or polyposis of the small intestine [8].

Although our patient was previously diagnosed with PJS, we confirmed 2 of the 3 criteria mentioned through physical examination and the imaging study findings, confirming the diagnosis of PeutzJeghers syndrome. Concerning the morphology, if polyps larger than $0.5 \mathrm{~mm}$ are detected, the recommended management is endoscopic polypectomy or double-balloon endoscopy to reduce the risk of complications $[9,10,12,13]$. The aforementioned is performed considering the inherent morbidity of a surgical procedure and the possibility of a residual short bowel syndrome due to resection of intestinal segments. However, if a large load of polyps is detected, the control by endoscopic approach is not achieved, or there is a complication, the most accepted procedure is a laparotomy with intraoperative enteroscopy since it offers direct vision to identify and resect the polyps [14]. In our case report, both approaches were performed. First, an endoscopic polypectomy was performed to treat the obstruction of the lumen, with the consequent GOO caused by a polyp located in the duodenum. Finally, a laparotomy was carried out with intraoperative enteroscopy due to the finding of multiple new polyps in monitoring exams and the slowing down of the intestinal transit associated with the patient's symptoms.

Patients with PJS may present complications such as intussusception, anemia, obstruction, perforation, and cancer, the latter being the most significant and studied given its relationship with PJS. However, given that biopsies did not show the presence of cancerous cells, this issue was not addressed.
In the literature, there is a lack of official epidemiological data on PJS complications. Nonetheless, according to the published case reports, intussusception is the most frequent complication (excluding cancer), which can cause intestinal obstruction when it is generated by large polyps ( $>15 \mathrm{~mm}$ ), being the small intestine the most affected site, specifically the jejunum [15]. On the other hand, intestinal obstruction, together with anemia, is the second most reported complication and is produced by the appearance of a large polyp that covers the entire intestinal lumen. Also, these complications may result from an associated intussusception (in the case of obstruction) and injury/bleeding of the polyp or cancer (in the case of anemia). There is evidence of PJS cases associated with other diseases and, therefore, presented in an atypical way such as i) an extra ampullary PJ polyp, which causes duodenal intussusception and presents with biliary obstruction and ii) a PJ polyp located in the appendix, presented with concomitant appendicitis or with melena as the only symptom.

Nevertheless, no cases of PJS have been reported associated with a concomitant GOO or intestinal aperistalsis. In this case report, the patient's clinical picture coincided with the GOO, where the polyps in the duodenum may act as a mechanical factor causing food retention and its related symptoms. In addition, it is important to highlight that the jejunal smooth muscle atrophy that the patient presented could play a role in a possible synergistic factor in the G0O. However, more studies are necessary to establish concrete and detailed causal relationships between both syndromes.

\section{Conclusion}

Peutz-Jeghers syndrome is a rare disease that has not been deeply studied. As far as we know, there are few reported cases of PJS concomitant with other syndromes, specifically gastric outlet obstruction. There is an undiscovered and/or studied relationship between these pathologies, especially considering the high probability that there are more cases globally, with these characteristics, without reporting. We speculate that the association of gastric outlet obstruction could be based on a mechanical effect generated by the polyps of the small intestine, which could alter the function of the stomach. This alteration could contribute to the aperistalsis of the jejunum, causing the symptomatology. Despite the above, more evidence is needed to demonstrate this association, mainly if muscular atrophy is part of the PJS.

\section{Conflict of Interest}

The authors declare that they have no conflict of interest.

\section{References}

1. Wu M, Krishnamurthy K (2020) Peutz-Jeghers Syndrome.

2. Idrogo Regalado B, Frisancho Velarde 0 (2016) Síndrome de PeutzJeghers. Presentación de cinco casos. Rev Gastroenterol Peru 36(2): 165-168 
3. McGarrity T, Amos C (2006) Peutz-Jeghers syndrome: clinicopathology and molecular alterations. Cellular and Molecular Life Sciences 63(18): 2135-2144.

4. Choudhury S, Das A, Misra P, Ray U, Sarangi S (2018) Peutz-Jeghers Syndrome: A Circumventable Emergency. Indian Journal of Dermatology 63(2): 168-171.

5. Calva D, Howe J (2008) Hamartomatous Polyposis Syndromes. Surgical Clinics of North America. 88(4): 779-817.

6. Tsao H (2000) Update on familial cancer syndromes and the skin. Journal of the American Academy of Dermatology 42(6): 939-969.

7. Peutz-Jeghers syndrome: Clinical manifestations, diagnosis, and management.

8. Kliegman R Nelson (2020) Tratado de Pediatría. pag 2061-2064, cap 372. Barcelona: Elsevier.

9. Gupta A, Postgate A, Burling D, Ilangovan R, Marshall M, et al. (2010) A Prospective Study of MR Enterography Versus Capsule Endoscopy for the Surveillance of Adult Patients With Peutz-Jeghers Syndrome. American Journal of Roentgenology 195(1): 108-116.

\section{ISSN: 2574-1241}

DOI: $10.26717 /$ BJSTR.2021.38.006160

Marcelo Zamorano, Duxan Arancibia. Biomed J Sci \& Tech Res

(C) (P) This work is licensed under Creative

Submission Link: https://biomedres.us/submit-manuscript.php
10. Tomas C (2014) Update on imaging of Peutz-Jeghers syndrome. World Journal of Gastroenterology 20(31): 10864

11. Gastroparesis: Etiology, clinical manifestations, and diagnosis.

12. Beggs A, Latchford A, Vasen H, Moslein G, Alonso A, et al. (2010) PeutzJeghers syndrome: a systematic review and recommendations for management. Gut 59(7): 975-986.

13. Syngal S, Brand R, Church J, Giardiello F, Hampel H, et al. (2015) ACG Clinical Guideline: Genetic Testing and Management of Hereditary Gastrointestinal Cancer Syndromes. American Journal of Gastroenterology. 110(2): 223-262

14. Hinds R, Philp C, Hyer W, Fell J (2004) Complications of Childhood Peutz-Jeghers Syndrome: Implications for Pediatric Screening. Journal of Pediatric Gastroenterology and Nutrition 39(2): 219-220.

15. Tavusbay C, Acar T, Kar H, Atahan K, Kamer E (2018) The patients with Peutz-Jeghers syndrome have a high risk of developing cancer. Turkish Journal of Surgery 34(2): 162-164.

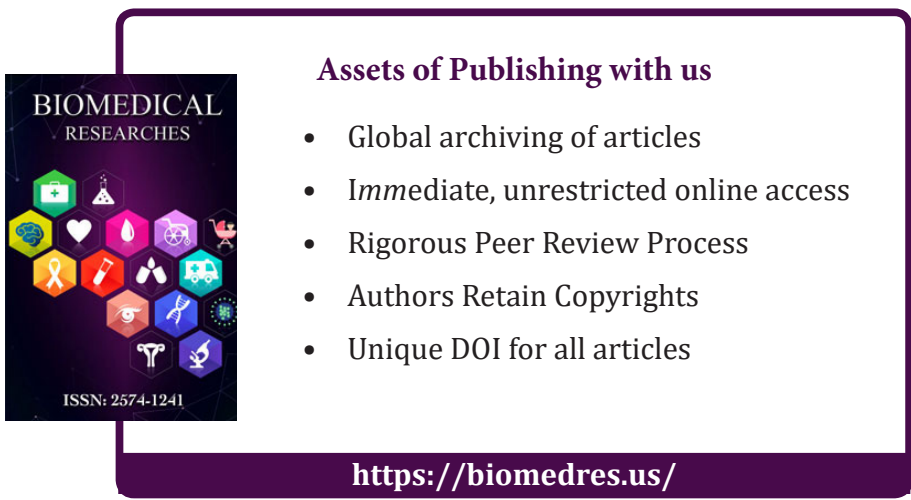

